Article

\title{
Comparing between Different Sets of Preprocessing, Classifiers, and Channels Selection Techniques to Optimise Motor Imagery Pattern Classification System from EEG Pattern Recognition
}

\author{
Francesco Ferracuti ${ }^{1}$ D , Sabrina Iarlori ${ }^{1}$, Zahra Mansour ${ }^{1}$, Andrea Monteriù ${ }^{1} \mathbb{D}$ and Camillo Porcaro ${ }^{2,3,4, *}$ \\ 1 Department of Information Engineering, Università Politecnica delle Marche, 60131 Ancona, Italy; \\ f.ferracuti@univpm.it (F.F.); s.iarlori@univpm.it (S.I.); zahramansour94@gmail.com (Z.M.); \\ a.monteriu@univpm.it (A.M.) \\ 2 Department of Neuroscience and Padova Neuroscience Center (PNC), University of Padova, \\ 35128 Padova, Italy \\ 3 Institute of Cognitive Sciences and Technologies (ISCT)—National Research Council (CNR), 00185 Rome, Italy \\ 4 Centre for Human Brain Health, School of Psychology, University of Birmingham, Birmingham B15 2TT, UK \\ * Correspondence: camillo.porcaro@unipd.it; Tel.: +39-0498212534
}

\section{check for}

updates

Citation: Ferracuti, F.; Iarlori, S.;

Mansour, Z.; Monteriù, A.; Porcaro,

C. Comparing between Different Sets of Preprocessing, Classifiers, and Channels Selection Techniques to Optimise Motor Imagery Pattern Classification System from EEG Pattern Recognition. Brain Sci. 2022, 12, 57. https://doi.org/10.3390/ brainsci12010057

Academic Editor: Ivan Volosyak

Received: 30 October 2021

Accepted: 23 December 2021

Published: 31 December 2021

Publisher's Note: MDPI stays neutral with regard to jurisdictional claims in published maps and institutional affiliations.

Copyright: (C) 2021 by the authors. Licensee MDPI, Basel, Switzerland. This article is an open access article distributed under the terms and conditions of the Creative Commons Attribution (CC BY) license (https:// creativecommons.org/licenses/by/ $4.0 /)$.

\begin{abstract}
The ability to control external devices through thought is increasingly becoming a reality. Human beings can use the electrical signals of their brain to interact or change the surrounding environment and more. The development of this technology called brain-computer interface (BCI) will increasingly allow people with motor disabilities to communicate or use assistive devices to walk, manipulate objects and communicate. Using data from the PhysioNet database, this study implemented a pattern classification system for use in a BCI on 109 healthy volunteers during real movement activities and motor imagery recorded by 64-channels electroencephalography (EEG) system. Different classifiers such as Support Vector Machine (SVM), K-Nearest Neighbors (KNN), and Decision Trees (TREE) were applied on different combinations of EEG channels. Starting from two channels (C3, C4 and CP3 and CP4) positioned on the contralateral and ipsilateral sensorimotor cortex, the Region of Interest (RoI) centred on C3/Cp3 and C4/Cp4 and, finally, a data-driven automatic channels selection was tested to explore the best channel combination able to increase the classification accuracy. The results showed that the proposed automatic channels selection was able to significantly improve the performance of each classifier achieving $98 \%$ of accuracy for classification of real and imagined hand movement (sensitivity $=97 \%$, specificity $=99 \%$, AUC $=0.99$ ) by SVM. While the accuracy of the classification between the imagery of hand and foot movements was $91 \%$ (sensitivity $=87 \%$, specificity $=86 \%$, AUC $=0.93$ ) also with SVM. In the proposed approach, the data-driven automatic channels selection outperforms classical a priori channel selection models such as $\mathrm{C} 3 / \mathrm{C} 4, \mathrm{Cp} 3 / \mathrm{Cp} 4$, or RoIs around those channels with the utmost accuracy to help remove the boundaries of human communication and improve the quality of life of people with disabilities.
\end{abstract}

Keywords: brain-computer interface (BCI); electroencephalography (EEG); Support Vector Machine (SVM); K-Nearest Neighbors (KNN); decision tree; imagination movement (IM)

\section{Introduction}

The number of people who suffer from temporal or permanent movement disabilities is enormously growing worldwide. It is estimated to range between $20-50$ million people depending on the World Health Organization (WHO) [1]. People with such motor deficiencies faced an encumbrance on performing their daily activities [2]. Therefore, many intelligent assistive systems have been developed to improve the Quality of Life (QoL) for people with movement disabilities by facilitating communication and creating a smart interactive environment [3]. One of the significant intelligent assistive systems depends on acquiring and transforming the activation of the Human Central Nervous System (CNS) 
into an artificial output that can be controlling an external device or lead a prosthesis: such a system is called Brain-Computer Interface (BCI) [4].

The research on BCI started in 1973 [5] when a group of scientists at the University of California proposed for the first time the $\mathrm{BCI}$ expression and launched the $\mathrm{BCI}$ challenge, which aims to control an external object using the brain signal recorded by Electroencephalography (EEG) [6]. After BCI was defined by Vidal [5], the first use of a frequency band in a BCI was presented in 1988 [7,8]. Later papers used beta and other bands. The 1988 work on BCI robot control explicitly uses [9] an artificial intelligence (AI) algorithm with machine learning (training) period and examination (testing) period. The development of the AI algorithms provided a learning pattern recognition approach [10], helping to increase the quantity and the quality of the $\mathrm{BCI}$ research in general and, in particular, on the Motor Imagery (MI). In this paradigm, the visuomotor imagery has been used to replace the real execution of the movement [11]. MI is a voluntarily generated signal and is dependent on the subject's intention that represents one of the most common paradigms because it can be used with stroke patients who suffer from movement disabilities. At the same time, their mental imagery can work properly [12].

Regardless of the application, the first step of any $\mathrm{BCI}$ system is the acquiring of the brain activity usually performed by the EEG system thanks to their portability, cost less, and its high temporal resolution, and non-invasivity [13-15]. On the other hand, its noninvasivity decreases the quality of the acquired signals that are corrupted by biological and non-biological artefacts [16-18]. Thereover, different preprocessing techniques have been proposed to increase the EEG signal-to-noise ratio (SNR) such as Independent Component Analysis (ICA) based algorithm both online [19-21] and offline [16,18]. However, this step is very time-consuming and ICA experts are needed to perform it. Thus, recently semiand full-automated systems have been developed to eliminate those artefacts from EEG recordings such as SASICA, namely Semi-Automatic Selection of Independent Components for Artifact [22].

The vital core for any pattern classification system used in a $\mathrm{BCI}$ is represented by translating the cleaned signal into a matrix of features. This can be carried out by investigating the EEG signal in the time domain and using a combination of EEG signals from all channels and calculating different values such as amplitude measurements [23], while other techniques are based on Power Spectrum Density (PSD) analysis on some specific frequency bands [24].

One of the most used feature in motor execution (ME) and MI is the Event-Related Desynchronisation and Synchronisation (ERD/ERS) [25-27] in the alpha band [8-13 Hz] and the beta bands [14-25 Hz] in the contralateral and ipsilateral sensorimotor areas [28,29]. ERD and ERS were introduced by Pfurtscheller in 1999 [27]. The ERD/ERS patterns showed high suitability in a pattern classification system implemented in a BCI, especially in the discrimination between right and left-hand movement. Moreover, they gave accuracy higher than $80 \%$ for online classification [30].

High-density EEG (hdEEG), on the one hand, gives higher spatial accuracy of the signal recorded [19], but, on the other hand, the large number of EEG channels presents a significant challenge in developing any BCI system [31]. Therefore, selecting the optimal channels combinations is one of the most active research fields because it has a massive effect on the computational load in pattern classification system performance and avoiding overfitting [32].

According to other papers as [33] that presents a new method for EEG channel selection optimisation based on relief function [34], the proposed approach aims to realise a datadriven procedure, considering every feature highly affected by channel selection and avoiding calculating the summation of features weight of each channel as in [33], in order to increase the accuracy performance.

In this study, we aim to create a pattern classification system for use in a BCI to classify between the real and imagined movement of the hands and foot on data from 109 volunteers taken from PhysioNet database [35] by computing and comparing pre- 
processed data throughout a semi-automatic ICA (saICA) [16,18] full automated ICA (SASICA) [22] and only filtered data. ERD features in different frequency bands, alpha [8-13 Hz], beta [14-25 Hz], and alpha plus beta [8-25 Hz] were extracted and different channel configurations, C3 and C4, Region of Interest (RoI) around C3 and C4, and optimised algorithm methods for channel selection were compared as well as different classifiers such as Support Vector Machine (SVM), K-Nearest Neighbors (KNN), and Decision Trees (TREE).

\section{Materials and Methods}

The data used in this work were obtained from the EEG Motor Movement/Imagery Dataset V 1.0.0 [35]. This dataset consists of EEG recordings from 109 participants involving 4 tasks and 14 experimental runs. Nine subjects were excluded from the database (subject Number: 38, 88, 89, 92, 93, 94, 100, 104 and 106) because their data contains incorrectly annotated errors labels. The participants performed the MI tasks, while a 64-channel EEG signal was recorded with a BCI2000 system [31] using the international 10-10 system with a $160 \mathrm{~Hz}$ sampling rate and an average reference. The MI is induced by a target that appears in one of four possible locations along the right edge of the screen. Then, a cursor appears at the left edge and moves from left to right at a constant rate with its vertical movement controlled by the power in an $\alpha$ or $\beta$ band at a location over sensorimotor cortex.

The participants performed 14 experimental runs: 2 baseline runs ( 1 with eyes open, 'run 1', and 1 with eyes closed, 'run 2', of 1 min each), and 3 runs for each of the following 4 task combinations ( 2 min each):

(i) Experimental runs 3, 7, 11. A target appears on either the left or the right side of the screen. The participant opens and closes the corresponding fist until the target disappears (Real Hand Movement "RHM"). Then the participant relaxes.

(ii) Experimental runs 4, 8, 12. A target appears on either the left or the right side of the screen. The participant imagines opening and closing the corresponding fist until the target disappears (Imagery Hand Movement "IHM"). Then the participant relaxes.

(iii) Experimental runs 5, 9, 13. A target appears on either the top or the bottom of the screen. Next, the participant opens and closes either fists (if the target is on top) and both feet (if the target is on the bottom) until the target disappears (Real Fists or Feet Movement "RFM"). Then the participant relaxes.

(iv) Experimental runs 6, 10, 14. A target appears on either the top or the bottom of the screen. The participant imagines opening and closing either both fists (if the target is on top) or both feet (if the target is on the bottom) until the target disappears (Imagery Fists or Feet Movement "IFM"). Then the participant relaxes.

In this study, we have studied four different combinations of all the tasks despite to concentrate only on the motor imagery movement as other papers did [36,37] as follow:

(i) Real Hand Movement (RHM) vs. Imagery Hand Movement (IHM): RHM vs. IHM;

(ii) Real Fists or Feet Movement (RFM) vs. Imagery Fists or Feet Movement (IFM): RFM vs. IFM;

(iii) Real Hand Movement (RHM) vs. Real Fists or Feet Movement (RFM): RHM vs. RFM;

(iv) Imagery Hand Movement (IHM) vs. Imagery Fists or Feet Movement (IFM): IHM vs. IFM;

\subsection{Pre-Processing}

The Dataset was imported into the EEGLAB v2019.1, where the signal of the three repetitions of each task and each subject were concatenated on one signal and decomposed into its main components using fastICA [38].

Three different techniques were applied and tested to improve the dataset's Signal to Noise Ratio (SNR) and to eliminate non-cerebral signals, i.e., eye movements, environmental and channel noise. The first technique is simply based on applying a bandpass filter [1-48 Hz], while the second technique relies on the auto selection and exclusion of the EEG noisy components using SASICA software [22] on the filtered data. Finally, the last technique used a semi-automatic ICA (saICA) based procedure $[16,18]$ to identify 
and classify artefactual components, where checking was applied to ensure no under or over-cleaning was reached by the saICA after filtering the data as for the previous method.

\subsection{Channels Selection}

In order to detect the optimal groups of channels that give the best performance of the model, various classification processes have been performed with different preclassification selected group of channels commonly used because placed on the region of motor cortex area [39]:

- $\quad$ C3/C4: using only the data from C3 and C4 channels.

- $\quad \mathrm{CP} 3 / \mathrm{CP} 4$ : using only the data from CP3 and CP4 channels.

- $\quad$ ROIC3/ROIC4: includes FC3, C5, C3, C1, CP3, FC4, C2, C4, C6, CP4.

- $\quad$ ROICp3/ROICp4: includes C3, CP5, CP3, CP1, P3, CP2, C4, CP6, P4 as is shown in Figure 1a-d, respectively.

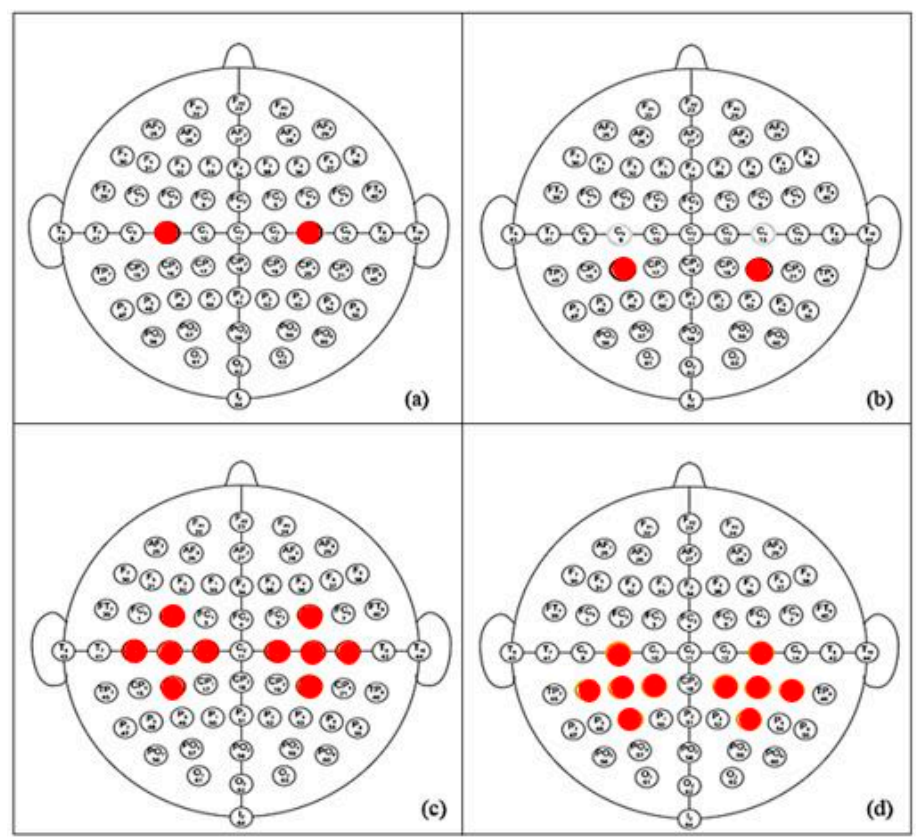

Figure 1. Different channels configuration tested: the red filled circles are located on the selected electrodes in the different configurations (a) C3/C4, (b) CP3/CP4, (c) ROI include C3/C4, and (d) ROI include $\mathrm{CP} 3 / \mathrm{CP} 4$.

\subsection{Features Extraction}

Event-Related Desynchronisation [27,40] in alpha, beta, and alpha plus beta was applied to the data (filter, SASICA, and saICA).

Three finite impulse response filters were used to compute the ERD in three different frequency bands and for each EEG channel, ERD_A (i.e., the desynchronisation value obtained integrating the alpha band between 8-13 Hz), ERD_B (i.e., the desynchronisation value obtained integrating the beta band between 14-25 Hz), and ERD_AB (i.e., the desynchronisation value obtained integrating the alpha lus band between 8-25 Hz).

ERD [27] was calculated using a pre-stimulus 'Rest' of 2 s (i.e., two seconds before the onset stimulus) and $2 \mathrm{~s}$ post-stimulus 'Task' (i.e., two seconds after the onset stimulus) across all trials and subjects. Once the two PSD (on the Rest and on the Task) were calculated, the ERD was abstained by the following formulation:

$$
\mathrm{ERD}=\frac{\sum_{\text {band }} P S D(\text { Task })-\sum_{\text {band }} P S D(\text { Rest })}{\sum_{\text {band }} P S D(\text { Rest })}
$$

'band' subscript refers to alpha, beta and alpha plus beta. 


\subsection{Classification}

The performance of three different classifiers was investigated, namely SVM, KNN, and TREE. SVM [41] is a supervised machine learning model that uses classification algorithms for two-group classification problems [42]. The second classifier is KNN [43], representing a non-parametric machine learning method where the input consists of the $\mathrm{k}$ closest training examples in feature space, while the output depends on whether KNN is used for classification or regression [44]. Finally, TREE classifier [45] was used as a predictive modelling approach. It uses a decision tree (as a predictive model) to go from observations about an item (represented in the branches) to conclusions about the target value of the item (represented in the leaves) [46].

The hyperparameters of each classifier have been optimised in a way that minimises five-fold cross-validation loss by using Bayesian optimisation [47].

\subsection{Optimisation Channels Selection}

A new optimisation method has been proposed in this study for data-driven channel selection. In contrast with the previous channel combinations which have been selected and fixed before the classification procedure [39] an optimisation system has been created to select the optimal combination of channels that gives the best model for each task.

This optimisation system initially works by computing the weight of each feature by using the relieff Matlab function, which calculates the Rank importance of predictors [34].

The descending order of the features based on their weight was given, then 64 groups of features were created, where the first group includes only the first feature in the feature order vector, while the second group includes the first and second features in the features order vector, and so on until the last group which includes all the features.

Later on, a 64-classification procedure will be performed using the 64 features group combination, and the combination of the features that give the highest accuracy was selected as the optimal group of channels. The previous procedure is explained in Figure 2.

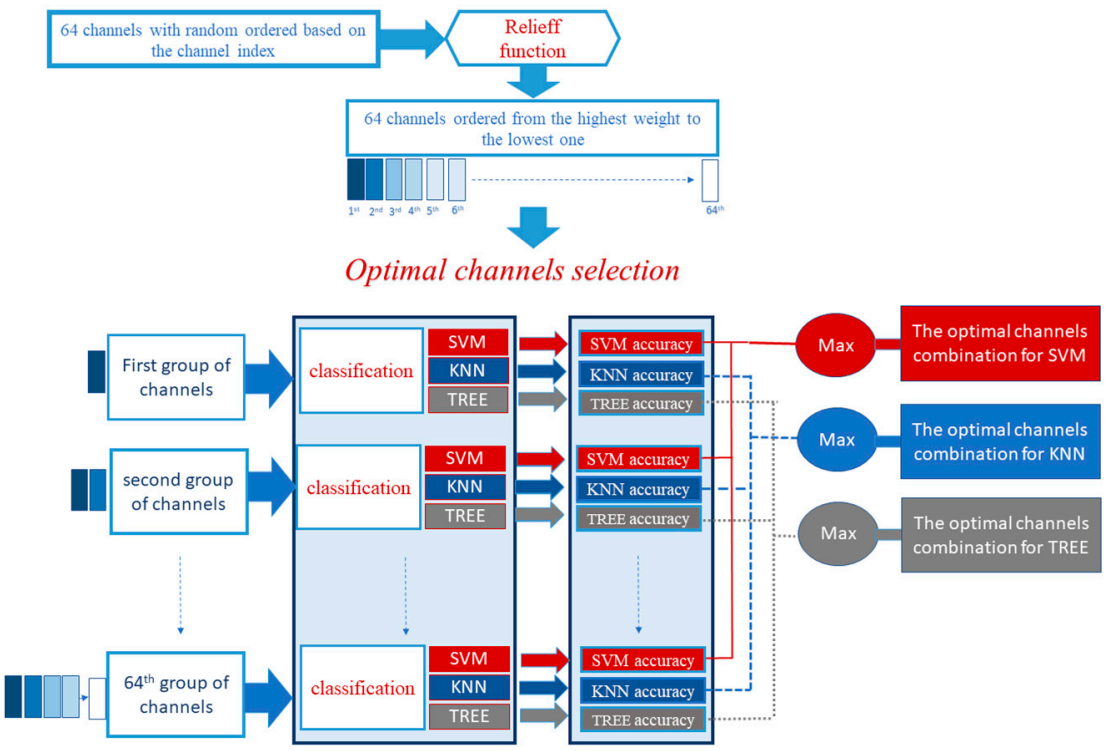

Figure 2. Schematic representation of the channel optimisation process and classification.

\section{Results}

\subsection{Comparison between the Cleaning Methods}

Three different cleaning techniques (Filter, SASICA, saICA) were applied separately on the EEG signal. Then the effect of the cleaning technique on the accuracy of the pattern classification system was calculated and tested by creating all the possible combinations 
with features (ERD_A, ERD_B, ERD_AB), classifiers, and channels selection technique proposed in this study. The accuracy was calculated following the formula:

$$
\text { Accuracy }=\frac{(\mathrm{TP}+\mathrm{TN})}{(\mathrm{TP}+\mathrm{TN}+\mathrm{FP}+\mathrm{FN})}
$$

where TP indicates the True Positive, TN the True Negative, FP the False Positive and FN the False Negative. The accuracy is obtained as the proportion of correct predictions (both true positives and negatives among the total number of cases examined.

The results with the highest accuracy have been obtained using the ERD_AB as a feature, the SVM as a classifier, and the optimal channels selection technique.

A significant difference in the accuracy value has been noticed based on the cleaning techniques among the combination of the different tasks (RHM vs. IHM, RFM vs. IFM, RHM vs. RFM, IHM vs. IFM).

The saICA technique showed the highest capability to clean the EEG signal without removing the important components. Thus, the data, cleaned with this method, give the highest accuracy across tasks comparison (mean $=94.25, \mathrm{SD}=3.86)$, followed by the data cleaned with SASICA (mean $=61.75, \mathrm{SD}=8.80$ ), while the filter application performs the lowest accuracy $($ mean $=53, \mathrm{SD}=8.67)$. Table 1.

The obtained accuracy using the three different cleaning techniques is summarised in

Table 1. Comparing the accuracy of classification using three different cleaning techniques (Filter, SASICA, and saICA) using ERD_AB as a feature, the optimal channels selection, and SVM classifier.

\begin{tabular}{cccccc}
\hline & RHM vs. IHM (\%) & RFM vs. IFM (\%) & RHM vs. RFM (\%) & IHM vs. IFM (\%) & $\begin{array}{c}\text { Mean } \pm \text { Standard } \\
\text { Deviation }\end{array}$ \\
\hline Filter & 45 & 61 & 60 & 46 & $53 \pm 8.67$ \\
SASICA & 68 & 50 & 60 & 69 & $61.75 \pm 8.80$ \\
saICA & 98 & 94 & 96 & 91 & $94.25 \pm 3.86$ \\
\hline
\end{tabular}

Thus, the results reported below from now on refer to the saICA cleaned data.

\subsection{General Comparison across Methods}

3.2.1. An Overview across All the Proposed Techniques

Several IM and RM EEG pattern classification methods have been created with different features (ERD_A, ERD_B, ERD_AB), several channel groups (C3/C4, CP3/CP4, ROI C3/C4, ROI CP3/CP4, and optimal channels) and classifiers (SVM, KNN, TREE). The performance has been tested by calculating the accuracy among four different tasks combinations (RHM vs. IHM, RFM vs. IFM, RHM vs. RFM, IHM vs. IFM). Table 2 summarises the classification accuracy among the different techniques.

Detailed analysis for each test is presented below.

The best results were obtained using ERD_AB as a feature and SVM as a classifier and the combination of the optimal channel (RHM vs. IHM $=98 \%$, RFM vs. IFM $=94 \%$, RHM vs. RFM $=96 \%$, and IHM vs. IFM =91\%). Extra analysis has been carried out in the following sections.

\subsubsection{Comparison between the Channel Selection Techniques}

Across all the tasks combinations, it has been found that the optimal channel selection techniques return the highest accuracy (mean $=94.75, \mathrm{SD}=1.49 \%$ ) followed by $\mathrm{ROI}$ $\mathrm{C} 3 / \mathrm{C} 4($ mean $=87.5, \mathrm{SD}=3.2 \%)$, ROI Cp3 $/ \mathrm{Cp} 4($ mean $=80.75, \mathrm{SD}=4.13 \%), \mathrm{C} 3 / \mathrm{C} 4$ (mean $=76, \mathrm{SD}=13.47 \%$ ) and the lowest values obtained using CP3 $/ \mathrm{CP} 4$ (mean $=69.75$, $\mathrm{SD}=7.22 \%$ ). Figure 3 shows the optimal channel selection for a particular combination of parameters (ERD_AB as feature and SVM as a classifier) and among the four different tasks combinations (RHM vs. IHM, RFM vs. IFM, RHM vs. RFM, IHM vs. IFM). As shown in 
meta-analysis by [48], many studies show recruitment of frontoparietal and central areas during the execution of the motor imagery of the lower and upper limbs.

Table 2. Summarise the classification accuracy using different sets of features (ERD_A, ERD_B, and ERD_AB) different sets of channel selection methods (C3/C4, CP3/CP4, ROI C3/C4, ROI CP3/CP4, and optimal channels) and different sets of classifiers (SVM, KNN, and TREE) to classify between different tasks combinations (RHM vs. IHM, RFM vs. IFM, RHM vs. RFM, and IHM vs. IFM).

\begin{tabular}{|c|c|c|c|c|c|c|c|c|c|c|c|c|c|}
\hline \multirow{2}{*}{$\begin{array}{l}\text { Channel } \\
\text { Selection }\end{array}$} & \multirow{2}{*}{ Features } & \multicolumn{3}{|c|}{ RHM vs. IHM (\%) } & \multicolumn{3}{|c|}{ RFM vs. IFM (\%) } & \multicolumn{3}{|c|}{ RHM vs. RFM (\%) } & \multicolumn{3}{|c|}{ IHM vs. IFM (\%) } \\
\hline & & SVM & KNN & TREE & SVM & KNN & TREE & SVM & KNN & TREE & SVM & KNN & TREE \\
\hline \multirow{3}{*}{ C3/C4 } & ERD_A & 50 & 51 & 52 & 59 & 57 & 55 & 62 & 61 & 60 & 56 & 57 & 54 \\
\hline & ERD_B & 72 & 72 & 70 & 61 & 63 & 62 & 65 & 63 & 58 & 58 & 60 & 56 \\
\hline & ERD_AB & 84 & 81 & 84 & 84 & 82 & 81 & 80 & 78 & 77 & 56 & 55 & 55 \\
\hline \multirow{3}{*}{$\mathrm{CP} 3 / \mathrm{CP} 4$} & ERD_A & 56 & 54 & 52 & 58 & 56 & 58 & 62 & 60 & 58 & 57 & 55 & 57 \\
\hline & ERD_B & 70 & 70 & 68 & 65 & 67 & 65 & 68 & 65 & 65 & 54 & 55 & 55 \\
\hline & ERD_AB & 83 & 82 & 80 & 61 & 60 & 60 & 81 & 78 & 75 & 54 & 54 & 55 \\
\hline \multirow{3}{*}{ ROI C3/C4 } & ERD_A & 78 & 81 & 61 & 83 & 79 & 61 & 87 & 88 & 67 & 79 & 68 & 60 \\
\hline & ERD_B & 92 & 78 & 74 & 86 & 77 & 69 & 96 & 82 & 78 & 75 & 68 & 58 \\
\hline & ERD_AB & 92 & 89 & 86 & 82 & 79 & 66 & 94 & 87 & 83 & 82 & 73 & 60 \\
\hline \multirow{3}{*}{$\begin{array}{c}\text { ROI } \\
\text { CP3/CP4 }\end{array}$} & ERD_A & 69 & 63 & 56 & 75 & 66 & 56 & 79 & 71 & 62 & 67 & 66 & 56 \\
\hline & ERD_B & 86 & 75 & 74 & 79 & 66 & 68 & 91 & 87 & 73 & 65 & 65 & 60 \\
\hline & ERD_AB & 93 & 90 & 82 & 77 & 71 & 61 & 78 & 77 & 60 & 75 & 71 & 68 \\
\hline \multirow{3}{*}{$\begin{array}{l}\text { Optimal } \\
\text { channels }\end{array}$} & ERD_A & 89 & 80 & 73 & 88 & 76 & 69 & 91 & 89 & 72 & 85 & 75 & 69 \\
\hline & ERD_B & 95 & 85 & 84 & 93 & 82 & 76 & 95 & 90 & 83 & 86 & 70 & 65 \\
\hline & ERD_AB & 98 & 88 & 93 & 94 & 85 & 87 & 96 & 88 & 91 & 91 & 90 & 68 \\
\hline
\end{tabular}

RHM/IHM

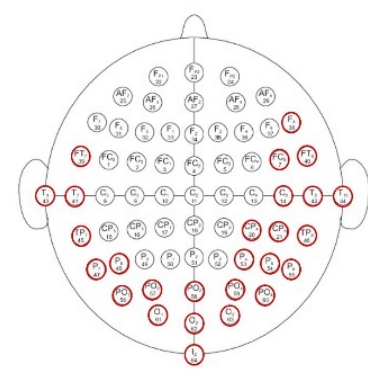

RHM/RFM

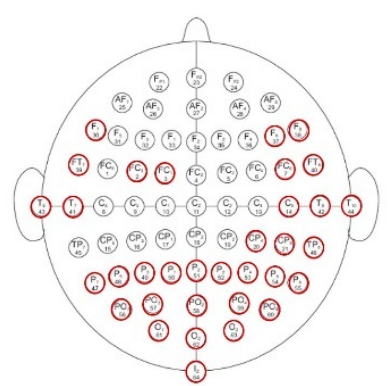

RFM/IFM

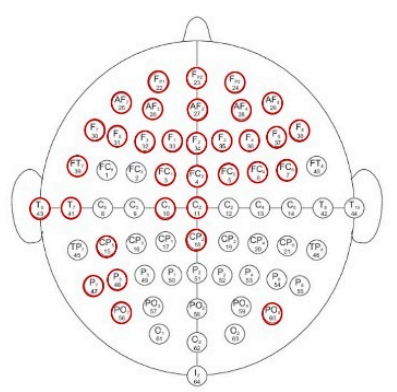

IHM/IFM

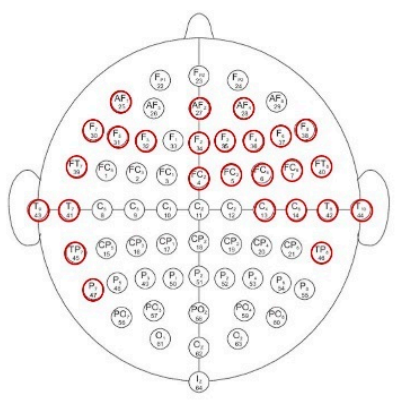

Figure 3. Optimal channel selection for a particular combination of parameters (ERD_AB as feature and SVM as a classifier) and among the four different combination tasks.

The classification accuracy of different BCIs was calculated by using ERD_AB as a feature and SVM as a classifier, and different sets of channels (C3/C4, CP3/CP4, ROI $\mathrm{C} 3 / \mathrm{C} 4$, ROI CP3/CP4, and optimal channels) to evaluate the performance of each group, as reported in Figure 4. 


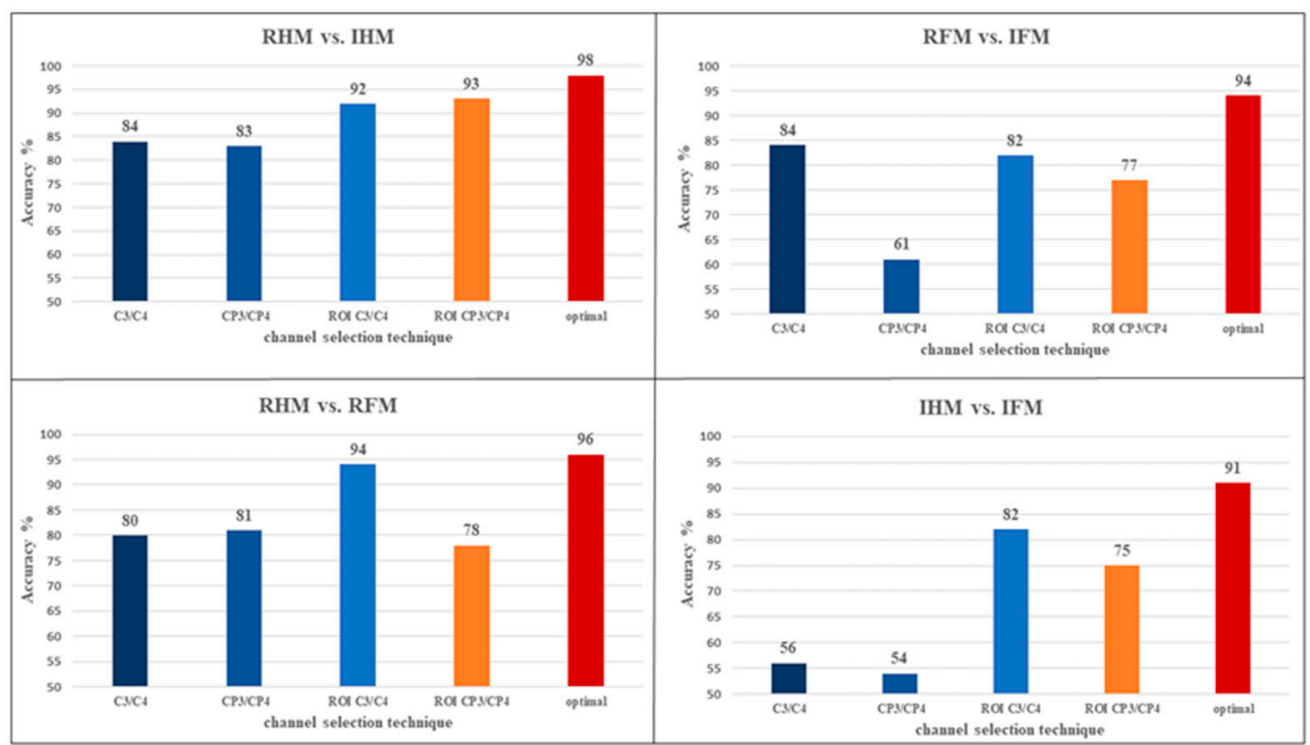

Figure 4. Column chart specifies the effect of the channel selection technique on the classification percentage accuracy to classify between different tasks. Showed that the highest accuracy is obtained by using the optimal channels selection technique. Using Semi-Auto ICA cleaned data and ERD_AB as a feature, SVM as a classifier.

\subsubsection{Comparison between the Features}

It has been found that the ERD_AB returns the highest accuracy across tasks comparisons $($ mean $=94.75 \pm \mathrm{SD}=1.49 \%)$ followed by ERD_B $($ mean $=92.25 \pm \mathrm{SD}=2.136 \%)$ and the lowest values obtained using ERD_A (mean $=88.25 \pm \mathrm{SD}=1.25 \%$ ).

The classification accuracy of different BCIs was calculated by using the optimal channels selection and SVM as a classifier and different sets of features (ERD_A, ERD_B, and ERD_AB) in order to evaluate the performance of each group, as reported in Figure 5.

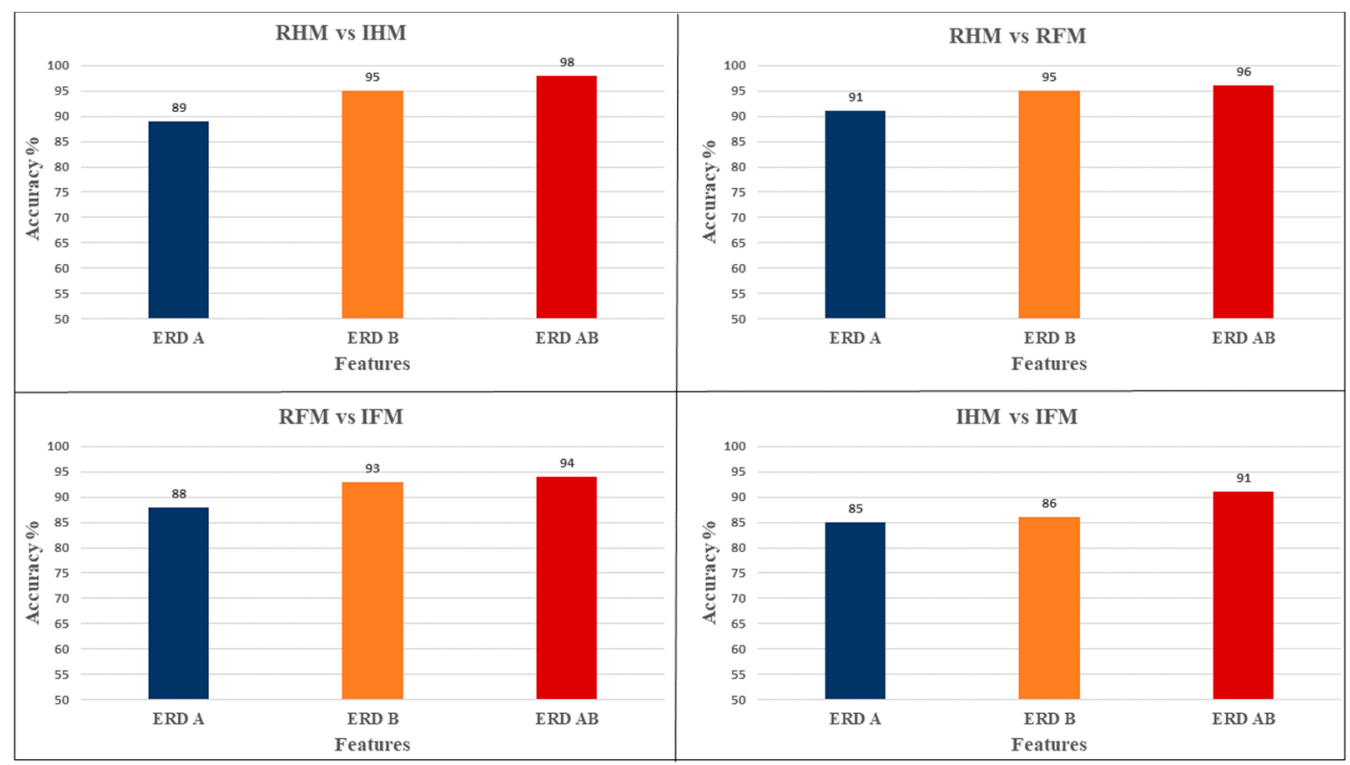

Figure 5. Column chart specifies the effect of the features on the classification percentage accuracy to classify between different tasks using semi-auto ICA cleaned data with the optimal channel selection and SVM as a classifier. Showed that the highest accuracy is obtained by using ERD_AB as a feature.

\subsubsection{Comparison between the Classifiers}

It has been found that the SVM returns the highest accuracy among all the tasks combinations $($ mean $=94.75 \pm \mathrm{SD}=1.49 \%)$ followed by KNN $($ mean $=87.75, \mathrm{SD}=1.031 \%)$ 
and the lowest values obtained using TREE (mean $=84.75, \mathrm{SD}=5.72 \%$ ). This result is explained in Table 3 which summarises the classification results.

Table 3. Classifiers mean accuracy among channels selection techniques, with respect to the features over the four tasks combinations.

\begin{tabular}{|c|c|c|c|c|c|c|c|c|c|c|c|c|c|}
\hline \multirow{2}{*}{ Channel Groups } & \multirow{2}{*}{ Features } & \multicolumn{3}{|c|}{ RHM vs. IHM (\%) } & \multicolumn{3}{|c|}{ RFM vs. IFM (\%) } & \multicolumn{3}{|c|}{ RHM vs. RFM (\%) } & \multicolumn{3}{|c|}{ IHM vs. IFM (\%) } \\
\hline & & SVM & KNN & TREE & SVM & KNN & TREE & SVM & KNN & TREE & SVM & KNN & TREE \\
\hline \multirow{3}{*}{$\begin{array}{c}\text { CP3/CP4, } \\
\text { ROI C3/C4, } \\
\text { ROI CP3/CP4, } \\
\text { Optimal channels }\end{array}$} & ERD_A & 68 & 66 & 59 & 73 & 67 & 60 & 76 & 74 & 64 & 69 & 64 & 59 \\
\hline & ERD_B & 83 & 76 & 74 & 77 & 71 & 68 & 83 & 77 & 71 & 68 & 64 & 59 \\
\hline & ERD_AB & 90 & 86 & 85 & 80 & 75 & 71 & 86 & 82 & 77 & 72 & 69 & 61 \\
\hline
\end{tabular}

The SVM classifier has always provided the best mean accuracy with respect to different single features as reported in Table 3. All the three classifiers achieve classification accuracy above $60 \%$ even when the features selected belong only to ERD_A and ERD_B set of features, but a clear increase is recorded with the SVM performance with respect to KNN and even more respect to TREE classifier.

The classification accuracy of different BCIs was calculated by using the optimal channels selection and ERD_AB as a feature and different sets of classifiers (SVM, KNN, and TREE) in order to evaluate the performance of each group, as reported in Figure 6.

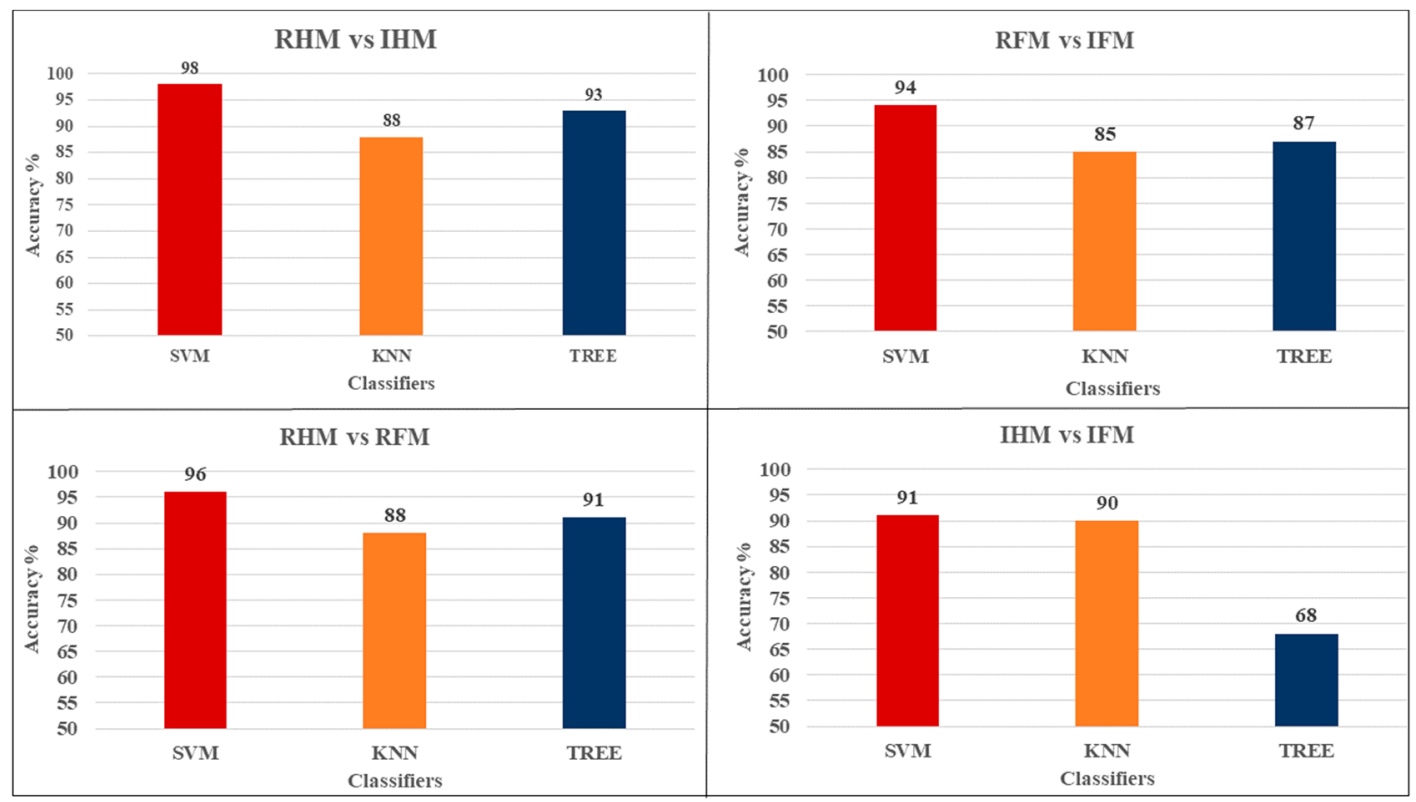

Figure 6. Column chart specifies the effect of the features on the classification accuracy to classify between different tasks using the optimal channels selection and ERD_AB as a feature. Showed that the highest accuracy is obtained by using the SVM classifier.

\subsection{Evaluation of the Optimal Channel Selection Technique}

In order to evaluate the performance of the proposed optimal channels selection technique (on ERD_AB as a feature and SVM as a classifier), several parameters (sensitivity, specificity, Area under the Curve (AUC), True Positive (TP), True Negative (TN), False Positive (FP), and False Negative (FN) have been calculated and summarised in Table 4. 
Table 4. Summarising the results obtained for the optimal channels selection technique showing the results obtained for the sensitivity, specificity, area under the curve AUC, true positive (TP), true negative (TN), false positive (FP) and false negative (FN) of a pattern classification system for use in a BCI using the semi-auto cleaned ICA data with the ERD_AB as a feature and SVM as a classifier.

\begin{tabular}{ccccc}
\hline & RHM vs. IHM & RFM vs. IFM & RHM vs. RFM & IHM vs. IFM \\
\hline Accuracy & $98 \%$ & $94 \%$ & $96 \%$ & $91 \%$ \\
Sensitivity & $97 \%$ & $92 \%$ & $91 \%$ & $87 \%$ \\
Specificity & $99 \%$ & $90 \%$ & $99 \%$ & $86 \%$ \\
AUC & 0.99 & 0.96 & 0.97 & 0.93 \\
TP & $194 / 200$ & $184 / 200$ & $181 / 200$ & $174 / 200$ \\
TN & $199 / 200$ & $181 / 200$ & $197 / 200$ & $171 / 200$ \\
FP & $1 / 200$ & $19 / 200$ & $3 / 200$ & $29 / 200$ \\
FN & $6 / 200$ & $16 / 200$ & $19 / 200$ & $26 / 200$ \\
\hline
\end{tabular}

The novel channels selection technique showed the highest fit with (RHM vs. IHM) task with Sensitivity $=97 \%$, specificity $=99 \%, \mathrm{AUC}=0.99, \mathrm{TP}=194 / 200, \mathrm{TN}=199 / 200$, $\mathrm{FP}=1 / 200$ and $\mathrm{FN}=6 / 200$.

\section{Discussion and Conclusions}

With the aim to define a pattern classification system for use in a BCI to classify between the real and the imagined movements, facilitating the interaction between people with limited motor abilities and their environment, a PhysioNet data set has been used [35], that includes EEG signals acquired during the performance of four different tasks: Real Hand Movement (RHM); Imagery Hand Movement (IHM); Real Fists or Feet Movement (RFM), and Imagery Fists or Feet Movement (IFM).

The goal of this study was to compare between different sets of pre-processing techniques (filtering, SASICA, and saICA), features (ERD in Alpha, Beta, and Alpha + Beta range), the different combinations of EEG channels (C3/C4, CP3/CP4, RoI centred on $\mathrm{C} 3 / \mathrm{C} 4$ and RoI centred on $\mathrm{CP} 3 / \mathrm{CP} 4$ and optimal channels combination), and different classifiers (SVM, KNN, and TREE) in order to evaluate their performance in a pattern classification system for use in a BCI.

The overall results have highlighted that the combination of the saICA as a cleaning method, the features ERD_AB, the optimal channel selection technique as the best channel configuration, and the SVM classifier give the best model solution, showing the highest accuracy values.

The different pre-processing approaches, based on filtering and independent component analysis methods, have been tested to reduce the signal-to-noise ratio. In this context, a semi-automated offline algorithm saICA [16,18] and a fully automated system SASICA [22] provided the best results with respect to the filtering data applying a pass-band filter followed by a notch filter.

The saICA outperforms the other methods because it controlled for over cleaning and down cleaning effects. In particular, while the filter approach probably suffers from an intrinsic down cleaning, keeping the motor artifacts that compromise the ERD feature extraction and consequently the classification, SASICA fully automatic version instead, over-cleaning the data, deletes some physiological activity during the task, compromising the feature extraction classification as well.

Moreover, regarding the necessity of cleaning the data, different pre-processing techniques, referred to supervised ICA approach, were highly time-consuming. On this aspect, neural networks can help since they do not need a strong pre-processing as shown in some studies [49]. In those studies, only minimal pre-processing such as removing or interpolating bad channels were used and all the rest was left to the burden of learning from a potentially noisy signal to the neural network [49]. On the other hand, machine learning methods based on artificial neural networks with the ability to use techniques that allow a system to automatically detect and classify features from raw data, including unsupervised 
training of raw input data (i.e., automatic feature selection and dimensionality reduction), are highly computationally expensive to train and to determine the hyperparameters. Consequently, the choice of the hyperparameters is mainly user-dependent [49].

The analysed EEG signal features have been the event-related desynchronisation ERD and synchronisation (ERS) in the alpha [8-13 Hz], beta [14-25 Hz], and alpha plus beta [8-25] bands, highly suitable for motor execution and MI. The ERD is the acquisition recording method to evoke through the visual stimulation, linked with motor-related brain functions, mainly execution and imagery of motor actions [50]. Two different types of ERD features can be differentiated: one short-lasting, localised into occipital areas and involving upper alpha components which most likely reflects primary visual processing and feature extraction, and the other longer-lasting, more widespread, prominent over parietal areas and maximal for lower-alpha components, mostly related to cognitive processing and mechanisms of attention [50]. Alpha ERD selectivity was mainly observed at motor areas, whereas beta ERD responses selective to motion obeying this law were recorded at motor and prefrontal nonmotor sites. Unilateral voluntary upper limb movement is accompanied by an ERD feature in the alpha and beta bands localised over the contralateral sensorimotor area [27]. The results related to ERD features within both frequency bands (alpha plus beta) were consistently stronger, arose faster, and more widespread while observing motion [51]. In our case, the best results obtained on alpha plus beta ERD support that both bands are involved during motor imagery but also during motor action since the best accuracy classification was obtained in those bands when considered together with respect to the only alpha or beta bands.

The test on different combinations of channels to detect the optimal groups of features returned the highest accuracy [52] on the classification percentage between the different tasks for the optimal channel selection techniques, with a mean of $94.75 \%$ accuracy applying the SVM classifier. It provided the best results with respect to single-channel selection, using only the data provided by channels C3 and C4 and CP3 and CP4, and ROI C3/C4 and ROI CP3/CP4. Using a single-channel approach might be a solution to avoid computational time according to [17]. In our opinion, the single-channel solution presents some issues in all the cases the task under investigation involves a long-range and distributed brain network [53]. Since the MI signal derives its activities from a distributed network [54], picking a single channel or an average of channels based on a topographic map could be misleading, especially if the aim is to describe the whole neuronal communication system $[49,52,53]$. Specifically, when an electrical potential is generated by a neuronal group, its activity is not only recorded from the electrode closest to this source but also from distant ones, due to the electric field propagation phenomenon. Consequently, each channel on the scalp derives its signal from more than one source. This problem worsens with the increasing number of sources activated at the same time. The generators of EEG activity cannot be reliably inferred based on a priori selected single channels, or a limited group of channels, due to the electric/magnetic field propagation problem. Moreover, using information coming from only one electrode can be misleading especially when the activated network is spread among the entire scalp [17,52]. In this regard, methods able to extract the under-investigated neural source by combining the activity from all the electrodes, are suitable for overcoming possible misleading results by avoiding the choice of a single electrode or averaging a group of electrodes. This should be the reason why the optimal channel selection has given the highest accuracy.

In addition, in the present study, the performance of three different classifiers SVM, KNN, and TREE were tested and compared at (i) the single feature set selected, (ii) concerning the channel group adopted, and (iii) for each of the four tasks analysed. After the optimisation of the hyperparameters for each classifier using the Bayesian optimisation approach [55] the best results were provided by the SVM classifier:

(i) even if all the three classifiers achieve classification accuracy above $60 \%$, a clear increase is recorded with the SVM also considering only ERD_A and ERD_B features (see Table 3); 
(ii) with respect to the channel group adopted SVM classifier rises always the higher mean value (see Table 5);

(iii) among all the tasks combination (mean $=94.75 \pm \mathrm{SD}=1.49 \%$ ). For each of the analysed single tasks and among the different combinations of channels group the SVM classifier has always provided the best mean accuracy with respect to different single features as reported in Table 2. Our results are in line with other literature on MI in BCI that support SVM as the best and most used classifier in this context $[56,57]$.

Table 5. Classifiers mean accuracy among tasks and selected features, with respect to channels selection techniques.

\begin{tabular}{|c|c|c|c|c|}
\hline \multirow{2}{*}{ Features } & \multirow[t]{2}{*}{ Channel Groups } & \multicolumn{3}{|c|}{$\begin{array}{l}\text { RHM vs. IHM (\%), RFM vs. IFM (\%), } \\
\text { RHM vs. RFM (\%), IHM vs. IFM (\%) }\end{array}$} \\
\hline & & SVM & KNN & TREE \\
\hline & $\mathrm{C} 3 / \mathrm{C} 4$ & 65.58 & 65 & 63.66 \\
\hline ERD_A & $\mathrm{CP} 3 / \mathrm{CP} 4$ & 64.08 & 63 & 62 \\
\hline ERD_B & ROI C3/C4 & 85.5 & 79 & 68.58 \\
\hline \multirow[t]{2}{*}{ ERD_AB } & ROI CP3/CP4 & 77.8 & 72 & 64 \\
\hline & Optimal channels & 91.75 & 83.16 & 77.5 \\
\hline
\end{tabular}

The aim of pooling features and testing these different combinations of task conditions, channels group, and classifiers was to investigate how their combination might improve the classification accuracy for a reliable MI-based BCI.

Finally, some consideration among the tasks classifying between RHM and IHM gives the highest accuracy $98 \%$, while comparing between IHM and IFM give the lowest accuracy $91 \%$ by using the same features and the same manner to select the channels (see Table 1). The better performance obtained compared between imagery tasks still represents a challenge, probably due to the higher variability between the performances among the volunteers and in particular, due to the absence of closed-loop feedback that might test the accuracy of the task performed by the volunteers.

To the best of our knowledge, we provide better overall classification results by comparing the results obtained with other studies working with the same data sets [58-61]. In particular, in Roots et al. [58], the $83.8 \%$ accuracy was obtained between the imagery movement task with the EEGNet Fusion, while in the proposed approach using ERD_AB and the optimal channels combinations with SVM classifier we reached $91 \%$ of the accuracy in the same task.

In other studies [59-61], that have used deep learning CNN (Convolutional Neural Network) to classify between events in IHM vs. IFM, obtained, respectively, $96 \%, 86 \%$, and $85.9 \%$ of accuracy but selecting only 10 subjects. The results presented in this study reached, instead, $91 \%$ of accuracy using all the subjects by applying the SVM as a classifier. Based on different time-domain features and classifiers but the same observed task, Alomari and colleagues [62] used Movement-Related Cortical Potentials (MRCP) and Neural Networks (NN) to classify between real and imagined hand movement have obtained an accuracy of $89 \%$ but involving only the first 6 subjects. Once again, in our study by using all the 100 subjects and combining ERD_AB as a feature and SVM classifier we raised accuracy equal to $98 \%$ by using the same tasks. Differently, Sleight and colleagues [63], used EEG averaged power and SVM to classify between RHM and IHM and they got accuracy equal to $69 \%$ with respect to our $98 \%$ of accuracy.

From the channels' combination point of view, this study proposed a new method to obtain the combination of the optimal channels. This method was able to obtain the highest accuracy compared to fixed channels selection. However, in some specific BCI cases, the lowest number of channels is mandatory and preferred. The RoI and the selected channels configurations are preferable related to physiological hypotheses, while multiple channels selected by data-driven approaches might provide increased accuracy results but less accurate from a physiological and anatomical perspective. For this reason, the number 
of channels and the way to select them might be a good compromise to be decided on the $\mathrm{BCI}$ application purpose.

Finally, from a computation point of view, the proposed model represents huge improvements in testing time, only $5 \mathrm{~ms}$ per sample, compared to the $107 \mathrm{~ms}$ when is used a convolutional neural network [58]. Moreover, even for the training time, this model requires just around $60 \mathrm{~min}$ to calculate the combination of the optimal channel for each feature and each classifier and to train the model against the days needed for training neural networks.

In conclusion, in this paper, we have presented a study based on data from the PhysioNet database. The automatically data-driven channel selection combined with an SVM classifier and a saICA as a pre-processing method has increased the classification accuracy for motor and imagery movement tasks to other approaches already presented in the literature.

\section{Open Challenges}

As a conclusion of our work, we provide a list of open challenges:

1. The use of different features such as Common Spatial Patterns (CSP) compared to ERD/ERS might be interesting to be tested.

2. The application of different feature ranking approaches might improve the accuracy and simultaneously lower the number of channels selected.

3. Different feature selection methods such as the Akaike Information Criterion (AIC) might guide the users to consider an optimal number of EEG channels.

4. Testing the accuracy of the single-subject performance instead of the accuracy at the group level.

Author Contributions: Conceptualization, C.P.; methodology, C.P. and Z.M.; software, C.P. and Z.M.; validation, C.P. and Z.M.; formal analysis, C.P. and Z.M.; writing - original draft preparation, C.P. and Z.M.; writing - review and editing, F.F., S.I., Z.M., A.M. and C.P; supervision, C.P.; project administration, C.P.; All authors have read and agreed to the published version of the manuscript.

Funding: This research received no external funding.

Institutional Review Board Statement: The study did not require any ethical approval.

Informed Consent Statement: The study used data from a dataset and does not involve humans directly.

Data Availability Statement: The study did not report any data.

Conflicts of Interest: The authors declare no conflict of interest.

\section{References}

1. World Health Organization. Summary World Report On Disability; World Health Organization: Geneva, Switzerland, 2011. Available online: https://www.who.int/teams/noncommunicable-diseases/sensory-functions-disability-and-rehabilitation/ world-report-on-disability (accessed on 29 October 2021).

2. T.E.C. to D. Studies. Disability and Development Report: Realizing the SDGs by, for and with Persons with Disabilities. 2019. Available online: w.un.org/development/desa/dspd/2019/04/un-disability-and-development-report-realizing-the-sdgs-by-for-andwith-persons-with-disabilities / (accessed on 29 October 2021).

3. Nemček, D. Quality of Life of People with Disabilities from Sport Participation Point of View. Acta Fac. Educ. Phys. Univ. Comen. 2016, 56, 77-92. [CrossRef]

4. Aboul Ella Hassanien, A.T.A. BCI: Current Trends and Applications; Springer International Publishing AG: Cham, Switzerland, 2015.

5. Vidal, J.J. Toward direct brain-computer communication. Annu. Rev. Biophys. Bioeng. 1973, 2, 157-180. [CrossRef] [PubMed]

6. Kübler, A. The history of BCI: From a vision for the future to real support for personhood in people with locked-in syndrome. Neuroethics 2020, 13, 163-180. [CrossRef]

7. Bozinovski, S.; Sestakov, M.; Bozinovska, L. Using EEG alpha rhythm to control a mobile robot. In Proceedings of the IEEE/Engineering in Medicine and Biology Society Annual Conference, New Orleans, LA, USA, 4-7 November 1988; Volume 10.

8. Bozinovski, S.; Bozinovska, L. Brain-Computer Interface in Europe: The thirtieth anniversary. Automatika 2019, 60, 36-47. [CrossRef]

9. Bozinovski, S. Mobile robot trajectory control: From fixed rails to direct bioelectric control. In Proceedings of the IEEE International Workshop on Intelligent Motion Control, IMC 1990, Istanbul, Turkey, 20-22 August 1990; Volume 2. 
10. Lance, B.J.; Kerick, S.E.; Ries, A.J.; Oie, K.S.; McDowell, K. Brain-computer interface technologies in the coming decades. Proc. IEEE 2012, 100, 1585-1599. [CrossRef]

11. Guger, C.; Kapeller, C.; Ortner, R.; Kamada, K. Motor imagery with brain-computer interface neurotechnology. In Motor Imagery: Emerging Practices, Role in Physical Therapy and Clinical Implications; Nova Science: New York, NY, USA, 2015.

12. Alimardani, M.; Nishio, S.; Ishiguro, H. Brain-Computer Interface and Motor Imagery Training: The Role of Visual Feedback and Embodiment. In Evolving BCI Therapy_Engaging Brain State Dynamics; Cognitive Science \& Artificial Intelligence IntechOpen: London, UK, 2018. Available online: https://books.google.it/books?hl=it\&lr=\&id=Jb-QDwAAQBAJ\&oi=fnd\&pg=PA73\& $\mathrm{dq}=$ Brain-Computer+Interface+and+Motor+Imagery+Training:+The+Role+of+Visual+Feedback+and+Embodiment\&ots $=$ -pjjDVLH4U\&sig=OfI7bW5F319TOW-6-c_Wg3zfaRM\#v=onepage\&q\&f=false (accessed on 29 October 2021).

13. Abiri, R.; Borhani, S.; Sellers, E.W.; Jiang, Y.; Zhao, X. A comprehensive review of EEG-based brain-computer interface paradigms. J. Neural Eng. 2019, 16, 011001. [CrossRef]

14. Steyrl, D.; Kobler, R.J.; Müller-Putz, G.R. On Similarities and Differences of Invasive and Non-Invasive Electrical Brain Signals in Brain-Computer Interfacing. J. Biomed. Sci. Eng. 2016, 9, 393-398. [CrossRef]

15. Volker, M.; Schirrmeister, R.T.; Fiederer, L.D.J.; Burgard, W.; Ball, T. Deep transfer learning for error decoding from noninvasive EEG. In Proceedings of the 2018 6th International Conference on Brain-Computer Interface, BCI 2018, GangWon, Korea, 15-17 January 2018; Volume 2018.

16. Barbati, G.; Porcaro, C.; Zappasodi, F.; Rossini, P.M.; Tecchio, F. Optimization of an independent component analysis approach for artifact identification and removal in magnetoencephalographic signals. Clin. Neurophysiol. 2004, 115, 1220-1232. [CrossRef] [PubMed]

17. Ferracuti, F.; Casadei, V.; Marcantoni, I.; Iarlori, S.; Burattini, L.; Monteriù, A.; Porcaro, C. A functional source separation algorithm to enhance error-related potentials monitoring in noninvasive brain-computer interface. Comput. Methods Programs Biomed. 2020, 191, 105419. [CrossRef]

18. Porcaro, C.; Medaglia, M.T.; Krott, A. Removing speech artifacts from electroencephalographic recordings during overt picture naming. Neuroimage 2015, 105, 171-180. [CrossRef]

19. Guarnieri, R.; Marino, M.; Barban, F.; Ganzetti, M.; Mantini, D. Online EEG artifact removal for BCI applications by adaptive spatial filtering. J. Neural Eng. 2018, 15, 056009. [CrossRef]

20. Hsu, S.H.; Mullen, T.R.; Jung, T.P.; Cauwenberghs, G. Real-Time Adaptive EEG Source Separation Using Online Recursive Independent Component Analysis. IEEE Trans. Neural Syst. Rehabil. Eng. 2016, 24, 309-319. [CrossRef]

21. Pion-Tonachini, L.; Hsu, S.H.; Chang, C.Y.; Jung, T.P.; Makeig, S. Online Automatic Artifact Rejection using the Real-time EEG Source-mapping Toolbox (REST). In Proceedings of the Annual International Conference of the IEEE Engineering in Medicine and Biology Society, EMBS, Honolulu, HI, USA, 18-21 July 2018; Volume 2018.

22. Chaumon, M.; Bishop, D.V.M.; Busch, N.A. A practical guide to the selection of independent components of the electroencephalogram for artifact correction. J. Neurosci. Methods 2015, 250, 47-63. [CrossRef]

23. Wei, Q.; Wang, Y.; Gao, X.; Gao, S. Amplitude and phase coupling measures for feature extraction in an EEG-based brain-computer interface. J. Neural Eng. 2007, 4, 120-129. [CrossRef]

24. Palaniappan, R. Brain computer interface design using band powers extracted during mental tasks. In Proceedings of the 2nd International IEEE EMBS Conference on Neural Engineering, Washington, DC, USA, 17-19 March 2005; Volume 2005.

25. Filho, A.C.S.S.; Simozo, F.H. XXVI Brazilian Congress on Biomedical Engineering. In Proceedings of the CBEB 2018, Armação de Buzios, RJ, Brazil, 21-25 October 2018; Costa-Felix, R., Machado, J.C., Alvaren, A.V., Eds.; Springer: Singapore, 2019.

26. Pfurtscheller, G.; Brunner, C.; Schlögl, A.; Lopes da Silva, F.H. Mu rhythm (de)synchronization and EEG single-trial classification of different motor imagery tasks. Neuroimage 2006, 31, 153-159. [CrossRef]

27. Pfurtscheller, G.; Lopes Da Silva, F.H. Event-related EEG/MEG synchronization and desynchronization: Basic principles. Clin. Neurophysiol. 1999, 110, 1842-1857. [CrossRef]

28. Aydemir, O.; Kayikcioglu, T. Wavelet transform based classification of invasive brain computer interface data. Radioengineering 2011, 20, 31-38.

29. Porcaro, C.; Mayhew, S.D.; Bagshaw, A.P. Role of the Ipsilateral Primary Motor Cortex in the Visuo-Motor Network during Fine Contractions and Accurate Performance. Int. J. Neural Syst. 2021, 31, 2150011. [CrossRef] [PubMed]

30. Thomas, E.; Fruitet, J.; Clerc, M. Investigating brief motor imagery for an ERD/ERS based BCI. In Proceedings of the Annual International Conference of the IEEE Engineering in Medicine and Biology Society, EMBS, San Diego, CA, USA, 28 August-1 September 2012.

31. Arvaneh, M.; Guan, C.; Ang, K.K.; Quek, C. Optimizing the channel selection and classification accuracy in EEG-based BCI. IEEE Trans. Biomed. Eng. 2011, 58, 1865-1873. [CrossRef]

32. Roy, S.; Rathee, D.; McCreadie, K.; Prasad, G. Channel Selection Improves MEG-based Brain-Computer Interface. In Proceedings of the International IEEE/EMBS Conference on Neural Engineering, NER, San Francisco, CA, USA, 20-23 March 2019; Volume 2019.

33. Liu, Q.; Liu, Y.; Chen, K.; Wang, L.; Li, Z.; Ai, Q.; Ma, L. Research on channel selection and multi-feature fusion of EEG signals for mental fatigue detection. Entropy 2021, 23, 457. [CrossRef] [PubMed]

34. Matlab Relieff Function Link. Available online: https://www.mathworks.com/help/stats/relieff.html (accessed on 29 October 2021)

35. Physionet Dataset. Available online: https://physionet.org/content/eegmmidb/1.0.0/ (accessed on 29 October 2021). 
36. Zebende, G.F.; Filho, F.M.O.; Cruz, J.A.L. Auto-correlation in the motor/imaginary human EEG signals: A vision about the FDFA fluctuations. PLoS ONE 2017, 12, e0183121. [CrossRef] [PubMed]

37. Valipour, S.; Shaligram, A.D.; Kulkarni, G.R. Spectral analysis of EEG signal for detection of alpha rhythm with open and closed eyes. Certif. Int. J. Eng. Innov. Technol. 2014, 9001, 1-4.

38. Hyvärinen, A. Fast and robust fixed-point algorithms for independent component analysis. IEEE Trans. Neural Netw. 1999, 10, 626-634. [CrossRef]

39. Ko, L.W.; Ranga, S.S.K.; Komarov, O.; Chen, C.C. Development of Single-Channel Hybrid BCI System Using Motor Imagery and SSVEP. J. Healthc. Eng. 2017, 2017, 3789386. [CrossRef]

40. Pfurtscheller, G.; Neuper, C.; Flotzinger, D.; Pregenzer, M. EEG-based discrimination between imagination of right and left hand movement. Electroencephalogr. Clin. Neurophysiol. 1997, 103, 642-651. [CrossRef]

41. Support Vector Machine Algorithm Matlab. Available online: https://www.mathworks.com/help/stats/support-vectormachines-for-binary-classification.html (accessed on 29 October 2021).

42. Costantini, G.; Todisco, M.; Casali, D.; Carota, M.; Saggio, G.; Bianchi, L.; Abbafati, M.; Quitadamo, L. SVM classification of EEG signals for brain computer interface. In Proceedings of the Frontiers in Artificial Intelligence and Applications, Salerno, Italy, 28-30 May 2009; Volume 204. Available online: https:/ / dl.acm.org/doi/10.5555/1671108.1671138 (accessed on 29 October 2021).

43. KNN Algorithm Matlab. Available online: https://www.mathworks.com/help/stats/classificationknn.html (accessed on 29 October 2021).

44. Md Isa, N.E.Z.; Amir, A.; Ilyas, M.Z.; Razalli, M.S. The Performance Analysis of K-Nearest Neighbors (K-NN) Algorithm for Motor Imagery Classification Based on EEG Signal. In Proceedings of the MATEC Web of Conferences, Sibiu, Romania, 7-9 June 2017; Volume 140.

45. Tree Classifier Matlab. Available online: https://www.mathworks.com/help/stats/decision-trees.html (accessed on 29 October 2021).

46. Arvaneh, M.; Guan, C.; Ang, K.K.; Quek, H.C. EEG channel selection using decision tree in brain-computer interface. In Proceedings of the APSIPA ASC 2010-Asia-Pacific Signal and Information Processing Association Annual Summit and Conference, Singapore, 14-17 December 2010.

47. Bashashati, H.; Ward, R.K.; Bashashati, A. Bayesian optimization of BCI parameters. In Proceedings of the Canadian Conference on Electrical and Computer Engineering, Vancouver, BC, Canada, 15-18 May 2016; Volume 2016.

48. Hétu, S.; Grégoire, M.; Saimpont, A.; Coll, M.P.; Eugène, F.; Michon, P.E.; Jackson, P.L. The neural network of motor imagery: An ALE meta-analysis. Neurosci. Biobehav. Rev. 2013, 37, 930-949. [CrossRef] [PubMed]

49. Alzahab, N.A.; Apollonio, L.; Di Iorio, A.; Alshalak, M.; Iarlori, S.; Ferracuti, F.; Monteriù, A.; Porcaro, C. Hybrid deep learning (Hdl)-based brain-computer interface (bci) systems: A systematic review. Brain Sci. 2021, 11, 75. [CrossRef]

50. Pfurtscheller, G.; Neuper, C.; Mohl, W. Event-related desynchronization (ERD) during visual processing. Int. J. Psychophysiol. 1994, 16, 147-153. [CrossRef]

51. Meirovitch, Y.; Harris, H.; Dayan, E.; Arieli, A.; Flash, T. Alpha and beta band event-related desynchronization reflects kinematic regularities. J. Neurosci. 2015, 35, 1627-1637. [CrossRef]

52. Porcaro, C.; Balsters, J.H.; Mantini, D.; Robertson, I.H.; Wenderoth, N. P3b amplitude as a signature of cognitive decline in the older population: An EEG study enhanced by Functional Source Separation. Neuroimage 2019, 184, 535-546. [CrossRef]

53. Mayhew, S.D.; Porcaro, C.; Tecchio, F.; Bagshaw, A.P. fMRI characterisation of widespread brain networks relevant for behavioural variability in fine hand motor control with and without visual feedback. Neuroimage 2017, 148, 330-342. [CrossRef]

54. Tecchio, F.; Porcaro, C.; Barbati, G.; Zappasodi, F. Functional source separation and hand cortical representation for a braincomputer interface feature extraction. J. Physiol. 2007, 580, 703-721. [CrossRef]

55. Bayesian Optimization Algorithm. Available online: https://www.mathworks.com/help/stats/bayesian-optimizationalgorithm.html (accessed on 29 October 2021).

56. Mebarkia, K.; Reffad, A. Multi optimized SVM classifiers for motor imagery left and right hand movement identification. Australas. Phys. Eng. Sci. Med. 2019, 42, 949-958. [CrossRef]

57. Ayatollahi, A. Classification of two motor imagery based on EEG signals in brain computer interface systems using LDA, SVM and GMM methods. In Proceedings of the 1st International Conference on Application of Research in Sciences and Engineering, Tehran, Iran, 27-28 April 2016.

58. Roots, K.; Muhammad, Y.; Muhammad, N. Fusion convolutional neural network for cross-subject eeg motor imagery classification. Computers 2020, 9, 72. [CrossRef]

59. Lun, X.; Yu, Z.; Chen, T.; Wang, F.; Hou, Y. A Simplified CNN Classification Method for MI-EEG via the Electrode Pairs Signals. Front. Hum. Neurosci. 2020, 14, 14. [CrossRef] [PubMed]

60. Dose, H.; Møller, J.S.; Iversen, H.K.; Puthusserypady, S. An end-to-end deep learning approach to MI-EEG signal classification for BCIs. Expert Syst. Appl. 2018, 114, 532-542. [CrossRef]

61. Karácsony, T.; Hansen, J.P.; Iversen, H.K.; Puthusserypady, S. Brain computer interface for neuro-rehabilitation with deep learning classification and virtual reality feedback. In Proceedings of the PervasiveHealth: Pervasive Computing Technologies for Healthcare, Reims, France, 11-12 March 2019. 
62. Alomari, M.H.; Samaha, A.; AlKamha, K. Automated Classification of L/R Hand Movement EEG Signals using Advanced Feature Extraction and Machine Learning. Int. J. Adv. Comput. Sci. Appl. 2013, 4, 4. [CrossRef]

63. Sleight, J.; Pillai, P.; Mohan, S. Classification of Executed and Imagined Motor Movement EEG Signals. 2009, pp. 1-10. Available online: https:/ / www.semanticscholar.org/paper/Classification-of-Executed-and-Imagined-Motor-EEG-Sleight-Pillai/8a9d0 ee78265cee260f1072f81f7819e0f752519\#citing-papers (accessed on 29 October 2021). 